demonstrated the specific modulation of several genes by direct effect of aPLs. In vivo Qred supplementation of APS patients for one month significantly improved the monocytes' atherothrombotic gene profile, reversing the altered expression of a number of genes related to thrombosis, atherosclerosis, inflammation, oxidative stress, and intracellular signalling

Conclusions: 1. Gene expression profile allowed the identification of relevant genes and pathways altered in monocytes of APS patients, which are associated with the pathogenesis of the disease and modulated, at least partially, by aPLs. 2 . We have branded novel and specific mRNAs related to CVD in APS monocytes, further modified by effect of in vivo Qred supplementation.

Acknowledgements: Funded by JA (CTS-7940) and Ministry of Health (ISCIII, PI15/01333 and RIER RD16/0012/0015) cofinanced with FEDER funds.

Disclosure of Interest: None declared

DOI: 10.1136/annrheumdis-2018-eular.6371

\section{FRI0268 GLUCOCORTICOID-INDUCED LEUCINE ZIPPER (GILZ) REPRESENTS A CHECKPOINT LIMITING TYPE I INTERFERON (IFN) PRODUCTION IN SLE}

C.T. Nataraja, E.F. Morand, J. Thomas, J. Harris, S.A. Jones. Department of Medicine, Monash University, Melbourne, Australia

Background: Glucocorticoids (GC) remain the mainstay of treatment in Systemic Lupus Erythematosus (SLE). Type I interferons (IFN), produced by plasmacytoid dendritic cells $(p D C)$ in response to Toll-Like receptors (TLR) ligands, are critical to SLE pathogenesis, but are not suppressed by GC. Glucocorticoid-Induced Leucine Zipper (GILZ) is an endogenous anti-inflammatory protein induced by GC. Beaulieu et al., 2010 However, whether GILZ regulates IFN production in SLE is not known.

Objectives: To test the hypothesis that GILZ inhibits the production of Type I IFN in SLE.

Methods: We performed in vitro analysis on $\mathrm{pDC}$ and bone marrow-derived $\mathrm{DC}$ (BMDC), and in vivo studies, of WT and GILZ-/- mice using stimuli of TLR7 (Imiquimod), TLR7/8 (Resiquimod) and TLR9 (CpG). IFN was measured using a IFN luciferase assay, and other cytokines with ELISA. IFN-stimulated gene signatures (ISG) were measured using qPCR. To determine whether GILZ regulates IFN in human SLE, we mined a public gene expression dataset GSE10325. Becker et al., 2013

Results: Deletion of GILZ resulted in excess pDC secretion of IFN in response to TLR7 $(p=0.0012)$ and TLR9 $(p=0.01)$ stimulation, and BMDC secretion of IFN, IL6 and TNF $\alpha$ in response to TLR7 $(p=0.0039,0.017,0.033), T L R 7 / 8(p=0.001$ $<0.0001,<0.0001)$ and TLR9 $(p=0.005,<0.0001,0.0034)$ stimulation respectively. Dexamethasone (DEX) induced GILZ in WT pDC and BMDC, and TLR stimulation suppressed GILZ expression in BMDC, but TLR-stimulated GILZ-/- cell failed to suppress IFN in response to DEX. Moreover, GILZ deficiency was associated with increased ISG in naïve spleen cells, naïve BMDCs and TLR7/9 stimulated pDC of GILZ-/- mice compared to WT mice. Correspondingly, increased IFN was seen in GILZ-/- mice in response to TLR7/8 stimulation in vivo. In GSE10325, we show that lower expression of GILZ was associated with high ISG (IFI44, IFI44L, RSAD2, IFI27) ( $\mathrm{p}=0.0021)$ in SLE patient peripheral blood B cells, and GILZ mRNA was negatively correlated with IFN signature $(r=-0.63, p=0.017)$ which in turn positively correlated with disease activity (SLEDAI2k) $(r=0.77, p=0.002)$.

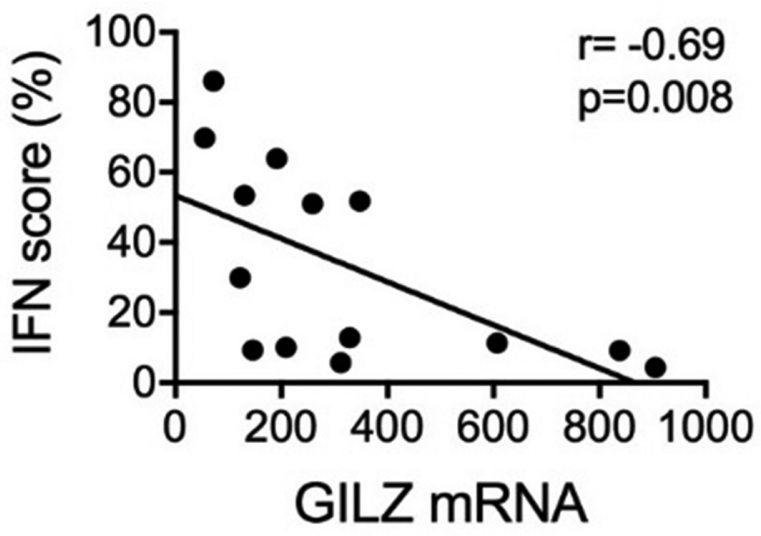

Conclusions: GILZ is an endogenous regulator of increased IFN production in response to TLR stimulation in vitro and in mice, and is negatively correlated with ISG in human SLE. This suggests that GILZ negatively regulates type I IFN production and GILZ based therapy may be a potential therapeutic strategy that could reduce steroid dependence in SLE.
REFERENCES:

[1] Beaulieu E, Ngo D, Santos L, Yang YH, Smith M, Jorgensen C, ... Morand EF. Glucocorticoid-induced leucine zipper is an endogenous antiinflammatory mediator in arthritis. Arthritis Rheum 2010;62(9):2651-2661. doi:10.1002/art.27566

[2] Becker AM, Dao KH, Han BK, Kornu R, Lakhanpal S, Mobley AB, Davis LS. SLE peripheral blood B cell, T cell and myeloid cell transcriptomes display unique profiles and each subset contributes to the interferon signature. PLoS One 2013;8(6):e67003. doi:10.1371/journal.pone.0067003

Acknowledgements: NHMRC

Disclosure of Interest: None declared

DOI: 10.1136/annrheumdis-2018-eular.1847

\section{FRI0269 DEFICIENCY OF GLUCOCORTICOID-INDUCED LEUCINE ZIPPER (GILZ) DISINHIBITS IFN PATHWAYS AND EXACERBATES NEPHRITIS IN THE LYN-DEFICIENT MURINE MODEL OF LUPUS}

C.T. Nataraja, E.F. Morand, J. Thomas, J. Harris, S.A. Jones. Department of Medicine, Monash University, Melbourne, Australia

Background: Systemic lupus erythematosus (SLE) is a multi-system autoim mune disease of unknown etiology. Lyn-deficient mice develop lupus-like autoimmunity due to hyperactive $B$ cells resulting in excess IL-6 production, and cyclical exacerbation of inflammation by further activation of B and T cells. Tsantikos et al., 2010 C57BL/6 mice deficient in glucocorticoid-induced leucine zipper (GILZ), an intracellular protein involved in glucocorticoid effects on immunity, develop lupus-like autoimmunity and excess B cell activation. Jones et al., 2016. However, the effect of GILZ in lupus-prone mice is unknown.

Objectives: We aim to test the hypothesis that GILZ modulates autoimmunity in the lyn deficient murine model of lupus.

Methods: We generated GILZ-deficient mice on a lyn-deficient background (GILZ-lyn double knock out (DKO)) and compared them to WT and lynKO mice. The effects of GILZ deficiency on spleen weight, nephritis, Type I interferoninduced genes (ISG) and autoantibodies were examined.

Results: We observed heightened lupus-like autoimmunity in GILZ-lyn DKO mice, compared to LynKO, that include increased spleen weights $(p=0.041)$ and more severe glomerulonephritis, especially segmental necrosis. A panel of ISG (ifi44, usp18, oas3, cxcl10, isg15, $m \times 1$, irf7, stat1 and ifit3) and an overall ISG score was significantly increased $(p=0.0023)$ in GILZ-lyn DKO mice compared to LynKO. In contrast, serum autoantibodies (dsDNA, Sm, histone, Jo-1, Scl-70, SSA, SSB, U1RNP, Ro52,) were not increased in GILZ-lyn DKO mice compared to LynKO mice.

\section{Glomerular segmental necrosis}

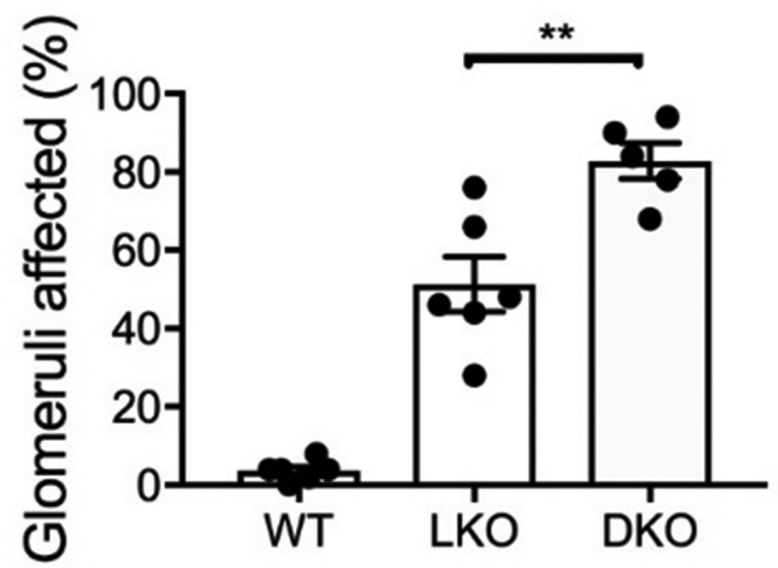

Conclusions: In LynKO lupus-prone mice, spleen weight, glomerulonephritis and ISG profiles were significantly exacerbated by GILZ deficiency, while autoantibody titres were unaffected. This suggests that endogenous GILZ exerts an inhibitory effect on IFN pathways, in this lupus model. Therefore, GILZ potentially regulates the cycle of inflammation in SLE by inhibiting IFN responses 
downstream of autoantibodies. A GILZ-based treatment could be a potential therapeutic strategy in SLE.

\section{REFERENCES:}

[1] Jones SA, Toh AE, Odobasic D, Oudin MA, Cheng Q, Lee JP, ... Morand EF. Glucocorticoid-induced leucine zipper (GILZ) inhibits B cell activation in systemic lupus erythematosus. Ann Rheum Dis, 2016;75(4):739-747. doi:10.1136/annrheumdis-2015-207744

[2] Tsantikos E, Oracki SA, Quilici C, Anderson GP, Tarlinton DM, Hibbs ML. Autoimmune disease in Lyn-deficient mice is dependent on an inflammatory environment established by IL-6. J Immunol 2010;184(3):1348-1360. doi:10.4049/jimmunol.0901878

Acknowledgements: NHMRC

Disclosure of Interest: None declared

DOI: 10.1136/annrheumdis-2018-eular.4094

\section{FRI0270 IDENTIFICATION OF NOVEL DYSREGULATED INTERFERON-INDUCIBLE NON-CODING RNAS IN SJÖGREN'S SYNDROME}

N. Means ${ }^{1,2}$, J.A. Ice ${ }^{1}$, I. Adrianto ${ }^{3}$, A.M. Stolarczyk ${ }^{1}$, M.L. Joachims ${ }^{1}$, A. Rasmussen ${ }^{1}$, J.M. Guthridge ${ }^{1}$, J.A. James ${ }^{1,2}$, R.H. Scofield ${ }^{1,2,4}$, K.L. Sivils ${ }^{1,2}$, C J. Lessard ${ }^{1,2}$. ${ }^{1}$ Oklahoma Medical Research Foundation; ${ }^{2}$ University of Oklahoma Health Sciences Center, Oklahoma City; ${ }^{3}$ Henry Ford Health System, MI;

${ }^{4}$ Department of Veterans Affairs Medical Center, Oklahoma City, USA

Background: Sjögren's syndrome (SS) is a chronic, heterogeneous disease with hallmark features of auto-inflammation and autoantibody production. Upregulation of type I and II interferon-stimulated genes (ISGs), known as the "Interferon (IFN) Signature" is correlated with anti-Ro titers and has been observed both in the salivary glands and peripheral blood of SS patients. Within the 2 p25.2 genomic interval, the long non-coding RNA (IncRNA) negative regulator of the interferon response (NRIR) has been identified as inducible by type I IFN and is responsible for the downregulation of the ISGs CMPK2 and RSAD2[.

Objectives: We sought to identify additional unannotated ISGs IncRNAs that are differentially expressed (DE) in SS patients utilising correlated expression of RSAD2.

Methods: We evaluated and compared the transcriptome of anti-Ro(+) patients $(n=27)$ and healthy controls $(n=27)$ using RNA-seq with DE defined as $\mathrm{q}<0.05$ and a fold change $(\mathrm{FC}) \geq 2$ or $\leq 0.5$. $\mathrm{qRT}$-PCR was used to validate $D E$ with primers specific for each RNA of interest. To understand the biological relevance of these transcripts, we performed in vitro time-course experiments to compare the transcriptional changes of unstimulated cells and cells stimulated with either PMA/ionomycin (PMA/I) or universal type I IFN for 36 hours measuring 7 time points.

Results: One of the most overexpressed type I ISGs in the SS Ro(+) is RSAD2 $(F C=8.05, p=3.29 \times 10 E-07)$. Because of its role in the type I IFN pathway, pairwise correlation coefficients between all DE transcripts and RSAD2 for SS Ro(+) patients were calculated. In total, we found 223 transcripts exhibiting correlation with RSAD2 expression (Pearson's $\mathrm{r}>+0.70$ or $<-0.60$ ), including NRIR (FC=2.72, $\mathrm{p}=5.87 \times 10 \mathrm{E}-03)$ and $C M P K 2(\mathrm{FC}=2.53, \mathrm{p}=3.58 \times 10 \mathrm{E}-03)$. Of the 223 transcripts, $14 \mathrm{DE}$ expressed IncRNAs correlated with RSAD2 expression. Several antisense ncRNAs situated nearby to other type I ISGs correlated with RSAD2, including: AC099063.1 ( $\mathrm{FC}=2.51), A C 004551.1$ ( $\mathrm{FC}=3.35)$, and AP001610.1 ( $\mathrm{FC}=4.12)$. We confirmed upregulation of these IncRNAs by qRT-PCR from the independent replication (14 $\mathrm{Ro}(+)$ and 36 controls) cohort $(\mathrm{p}=2.49 \mathrm{X} 10 \mathrm{E}-02,8.63 \times 10 \mathrm{E}-06$, $1.17 \times 10 \mathrm{E}-04$, respectively). Based on the locations of the IncRNAs to type I ISGs, HSB-2 cells were stimulated with PMA/I or universal type I IFN. Using qRT$\mathrm{PCR}$ to measure the protein coding genes MX1, OAS1, and GBP5 along with the IncRNAs, AC099063.1 (GBP5-AS1), AP001610.1 (MX1-AS1), and AC004551.1 (OAS123-AS1) showed coordinated regulation with their protein coding counterparts with both stimuli.

Conclusions: Given the importance of the IFN signature to disease pathogenesis in the autoantibody positive patients, it is critical that we better understand how this complex pathway is coordinately regulated. Since one critical function of IncRNAs is to regulate the genome, characterising the mechanisms by which these 14 identified by this study regulate the ISG coordinately could result in new diagnostic and/or therapeutic options.

\section{REFERENCE:}

[1] Kambara H, et al. Nucleic Acids Res 2014;42(16):10668-80.

Disclosure of Interest: None declared

DOI: 10.1136/annrheumdis-2018-eular.4837

\section{FRI0271}

\section{DIMETHYL FUMARATE INHIBITS UBE2L3 MEDIATED} TLR7 SIGNALLING AND AUTOREACTIVE B CELL DEVELOPMENT IN SLE

D. Mauro ${ }^{1}$, V. Tsang ${ }^{1}$, I. Lucey-Clayton ${ }^{1}$, F. Rivellese ${ }^{1}$, S. Pagani ${ }^{1}$, F. Alam ${ }^{1}$ E. Pontarini ${ }^{1}$, A. Nerviani ${ }^{1}$, A. Pakozdi ${ }^{2}$, R. Rajakariah ${ }^{2}$, D. Pyne ${ }^{2}$, T.J. Vyse ${ }^{3}$ C. Pitzalis ${ }^{1}$, M.J. Lewis ${ }^{1} .^{1}$ Experimental Medicine and Rheumatology, Queen Mary University of London; ${ }^{2}$ Rheumatology Department, Mile End Hospital, Barts Health NHS Trust, ${ }^{3}$ Medical and Molecular Genetics, King's College London, London, UK

Background: Genetic studies have identified a single UBE2L3 risk haplotype which is associated with SLE and multiple autoimmune diseases, and leads to increased expression of UBE2L3 in EQTL studies. The E2 ubiquitin-conjugating enzyme UBE2L3 regulates $\mathrm{NF}-\kappa \mathrm{B}$ activation through regulation of the Linear Ubiquitination Chain Assembly Complex (LUBAC). Thus UBE2L3 regulates CD40-driven B cell activation. The UBE2L3 risk allele correlates with circulating plasmablast and plasma cell expansion in SLE individuals.

Objectives: To determine the effect of UBE2L3 and linear ubiquitination on TLR7 signalling, and test the effect of Dimethyl Fumarate (DMF), which has recently been shown to inhibit UBE2L3, on B cell and plasmablast differentiation in SLE. Methods: Confocal microscopy, immunoprecipitation and western blot were used to assess linear ubiquitin chain accumulation in TLR7-HEK293 cells after TLR stimulation by Resiquimod. The effect of UBE2L3 and LUBAC on TLR7 sig nalling was dissected by the use pf UBE2L3/LUBAC overexpression, dominant negative mutants and shRNA silencing, measuring NF-kB reporter activity, IkappaBalpha phosphorylation, gene expression by GPCR and IL-8 secretion. DMF was administered in vitro to human $B$ cells isolated from SLE patients $(n=15)$ and controls and cultured with Resiquimod and/or IFN $\alpha$ for 5-7 days. B cell viability/ proliferation, plasmablast differentiation were analysed by 10 -colour flow cytometry. Supernatants were assayed for immunoglobulin secretion and autoantibody production.

Results: TLR7 stimulation led to intracellular accumulation of linear ubiquitin chain comparable to TNF $\alpha$. UBE2L3 and LUBAC co-overexpression enhanced TLR7 driven NF-kB activation and led to increased NF- $\kappa B$ target mRNA expression and increased secretion of IL-8. The effect was specific to UBE2L3 compared to other E2 enzymes. Dominant negative mutant UBE2L3(C86S) or HOIP (C885S) or UBE2L3/HOIP shRNA suppressed the response to TLR7 stimulation. DMF showed a dose-dependent inhibition of TLR7-mediated NF-kB activation. In primary SLE and healthy B cells, DMF suppressed proliferation of switched and unswitched CD27 +memory B cells and blocked plasmablast differentiation. DMF profoundly inhibited immunoglobulin secretion and anti-nuclear autoantibodies production in response to TLR7 and IFNa stimulus.

Conclusions: Our data demonstrate that linear ubiquitination and UBE2L3 regulate TLR7 activation of NF- $\mathrm{KB}$. UBE2L3 silencing or pharmacological antagonism of UBE2L3 by DMF suppressed the response to TLR7 activation. Excessive TLR7 signalling has been linked to SLE development and enhanced B cell autoreactivity. Thus our data identify a novel mechanism by which the UBE2L3 risk haplotype contributes to SLE susceptibility. DMF suppressed plasmablast differentiation and inhibited TLR7 and IFNa induced autoantibody production. These results support a role for repositioning DMF (currently used to treat multiple sclerosis) in the treatment of SLE.

Disclosure of Interest: None declared

DOI: 10.1136/annrheumdis-2018-eular.6960

\section{FRI0272 PHARMACODYNAMIC EFFECTS OF ATACICEPT TREATMENT IN A CYNOMOLGUS MONKEY KLH ANTIGEN CHALLENGE MODEL}

E. Samy ${ }^{1}$, A. Bender ${ }^{1}$, Y. Wu ${ }^{1}$, V. Castagna ${ }^{2}$, E. Bertotti ${ }^{2}$, R. Boggio ${ }^{2}$, A. Paoletti $^{2}$, S. Riva ${ }^{2}$, P. Schneider ${ }^{3}$, P. Haselmayer ${ }^{4}$, J. DeMartino ${ }^{1}{ }^{1} E M D$ Serono Research and Development Institute, Inc. (a business of Merck KGaA, Darmstadt, Germany), Billerica, USA; ${ }^{2}$ Istituto di Ricerche Biomediche Antoine Marxer RBM SpA (an affiliate of Merck KGgA, Darmstadt, Germany), Colleretto Giacosa, Italy,

${ }^{3}$ Department of Biochemistry, University of Lausanne, Lausanne, Switzerland; ${ }^{4}$ Merck KGaA, Darmstadt, Germany

Background: Atacicept is an antagonist of the B cell regulatory factors BLyS (B lymphocyte stimulator) and APRIL (a proliferation inducing ligand). It is capable of binding to all known conformations of BLyS and APRIL and is thus expected to modulate the maturation, differentiation, and effector function of $B$ cells. We aimed to define the relationship between atacicept drug exposure and pharmacody namic (PD) effects in vivo. Here, we report results of our study examining the effects of atacicept on the immune response in a keyhole limpet hemocyanin $(\mathrm{KLH})$ challenge model in cynomolgus monkeys.

Methods: Cynomolgus monkeys (Macaca fascicularis) were injected with KLH on Day -33 , and on Day 1 received the first dose of either atacicept $(0.3,3$, or $30 \mathrm{mg} / \mathrm{kg}$ ) or vehicle followed by a KLH challenge. Animals were dosed weekly (Day 1, 8, 15) and were subsequently monitored for 2 weeks before the study ended on Day 29. Clinical signs, total and free drug levels, peripheral blood B and 